\title{
EDITORIAL Relapse of acute leukemia after a second allogeneic stem- cell transplantation; Is there any hope for cure?
}

(c) The Author(s), under exclusive licence to Springer Nature Limited 2021

Bone Marrow Transplantation (2022) 57:336-337; https://doi.org/ 10.1038/s41409-021-01508-2

Allogeneic stem-cell transplantation (SCT) is a curative therapeutic approach for acute leukemia. New treatment modalities led to marked reduction of non-relapse mortality after SCT in more recent years, however, recurrent diseases remains the major cause of treatment failure [1]. There is no standard of care for the treatment of relapse after SCT. Treatment goals and approaches vary from palliative care alone, to low-dose or intensive chemotherapy, targeted therapies (such as hypomethylating agents, tyrosine kinase inhibitors and various immunotherapies) and up to cellular therapies including donor lymphocyte infusions (DLI) or a second transplant, or any combinations [2]. Prior studies have shown that cellular therapy is required to achieve long-term remission or even possible cure [3]. A second transplant has an established role in this setting with about $20-25 \%$ of recipients achieving long-term disease control [4-6]. Achieving a second remission prior to second transplant and a long prior remission (>6-12 months) are considered the most significant factors predicting subsequent survival. However, there is no data to support a second transplant over DLI [7]. Relapse is the major obstacle after a second transplant occurring in more than $50 \%$ of recipients. There is much less data on treatment approaches in this setting and whether there is any hope for long- term survival or cure. In particular, the role of a third transplant has not been defined.

In this issue of Bone Marrow Transplant Kobayashi and coauthors report the largest series published so far, of 253 patients with relapsed/ refractory acute leukemia given a third allogeneic transplant and reported to the Transplant Registry Unified Management Program (TRUMP) in Japan [8]. With a median follow-up of 26 months, 29 patients are alive. The 3-year leukemia- free survival (LFS) and overall survival (OS) rates were $9.7 \%$ and $10.9 \%$, respectively. Similarly to the experience with a second transplant, a long remission after the second transplant ( $>2$ years), complete remission at the time of third transplant and good performance status were associated with better survival. Good risk patients had an expected 3-year OS of 36\%. The authors conclude that while prognosis is unfavorable, considering the lack of other option a third transplant can be considered in a subset of patients with a small chance for long- term survival.

Rank and colleagues recently reported the experience of the European Society for Blood and Marrow Transplantation (EBMT) in a third allogneic transplant in a series of 45 patients with acute leukemia, relapsing after a second transplant [9]. The 1- year LFS and OS were 11 and 20\%, respectively. Interestingly, there were no survivors among patients with acute lymphoblastic leukemia (ALL).

While the data are limited several question merit discussion. What is the overall impact of a third transplant on relapse after a second transplant? Are there other options and who should be a candidate for a third transplant? Who should be the third donor? What stem cell source, conditioning regimen and post-transplant therapy should be used?

The group of patients given a third transplant is obviously a very selected group. It was estimated to be 16 and $4.5 \%$ of all patients relapsing after a second transplant in the Japanese and EBMT studies, respectively. Assuming a cure rate of $10 \%$, this will allow cure of less than $1-2 \%$ of all patients relapsing after a second transplant. The data on a third transplant is so far retrospective and does not compare the transplant group to patients treated with other modalities. We have not offered a third transplant and among 47 patients relapsing after a second transplant, 3 patients were alive 5 years after relapse, 2 with no recurrent disease (personal communication). There are novel therapies that were not available during the two studies period. In ALL, new monoclonal antibodies and CAR-T cells are promising compared to the very few survivors of a third transplant in this disease. In Acute myeloid leukemia new treatment combinations such as azacitidine with venetoclax, new FLT3 inhibitors and IDH inhibitors, can achieve responses. While they are not considered curative they can extend survival. They may also be used for bridging to a third transplant. In all, it seems that young patients with a good performance status, long remission after a second transplant and achieving a good response, preferentially with negative measurable residual disease, can be candidates for a third transplants. Among other subsets, the chances for cure are minimal.

Theoretically, changing the donor in a subsequent transplant may reduce relapse by changing the graft-versus-leukemia (GvL) effect induced by minor histocompatibility antigens or by major HLA mismatches such as when using a mismatched haploidentical or umbilical cord blood donors. In the mismatched setting relapse after a previous transplant is often related to immune escape by the loss of the unshared haplotype [10]. Therefore, switching to a different donor is logical. However, in the setting of a second transplant, there is no proven survival advantage for donor or stem cell source change $[4,5,11]$. In the current study, $83 \%$ had a new donor for the third transplant and a donor change was associated with reduced relapse rate after the third transplant. Similarly, in the EBMT study $2 / 3$ of patients had at least 2 different donors in the three transplants and 56\% changed a donor between the second and third transplant. These patients seemed to have a better LFS. The majority of long term survivors in this study had a different donor that was unrelated or mismatched. It seems that after failure of 2 transplants a new donor is mandatory. Leukemia control is critical and every effort is needed to augment the GvL effect. In the Japanese registry, the vast majority of transplants were of umbilical cord or haploidentical donors. In these setting a new donor is absolutely required due to donor availability and the risk of immune escape, respectively. These types of donor source are also more rapidly available and the window of opportunities for a third transplant 
after achieving remission following relapse after a second transplant may be particularly short.

Non-relapse mortality is exceptionally high after a third transplant in such heavily pre-treated patients, ranging from 33 to $42 \%$ in the 2 studies. In particular, organ toxicities related to endothelial syndromes such as veno-occlusive disease of the liver and thrombotic microangiopathy may be much more common [8]. Therefore, reduced-intensity conditioning is very often used in this setting.

Many patients have already had prior GVHD. In the current study acute GVHD after the second transplant was associated with lower relapse after the third [8]. In other studies, chronic GVHD after the first transplant predicted lower rates of relapse after the second [7, 11]. The GvL effect either carries on to subsequent therapies or a supportive cytokine or allo-immune milieu induced by prior GVHD continues to affect donor cells even from a different source. However, there are no established data or guidelines on how to use data on prior GVHD in the selection of new donors. Chronic GVHD after subsequent transplant may reduce relapse rates. However, relapse rate after a third transplant is still very high, $47-57 \%$ in the 2 studies. Therefore, maintenance therapies are particularly required when available in this setting.

In conclusion, relapse after a second SCT carries a dismal outcome. However, there is some hope for selected subsets of patients for long-term survival and even potential cure. Novel therapies may achieve response and extend survival. A third transplant is feasible in a minority of patients and can be successful in young patients with good performance status, long remission after the second transplant, achieving a good remission prior to the third transplant, and possibly with changing to a different donor.

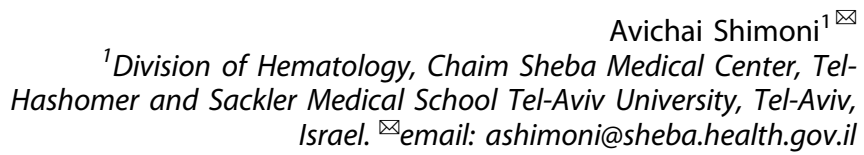

\section{REFERENCES}

1. Gooley TA, Chien JW, Pergam SA, Hingorani S, Sorror ML, Boeckh $M$, et al. Reduced mortality after allogeneic hematopoietic-cell transplantation. N Engl J Med. 2010:363:2091-101.

2. de Lima M, Porter DL, Battiwalla M, Bishop MR, Giralt SA, Hardy NM, et al. Proceedings from the National Cancer Institute's Second International Workshop on the Biology, Prevention, and Treatment of Relapse After Hematopoietic Stem Cell Transplantation: part III. Prevention and treatment of relapse after allogeneic transplantation. Biol Blood Marrow Transpl. 2014;20:4-13.

3. Schmid C, Labopin M, Nagler A, Niederwieser D, Castagna L, Tabrizi R, et al. Treatment, risk factors, and outcome of adults with relapsed AML after reduced intensity conditioning for allogeneic stem cell transplantation. Blood. 2012;119:1599-606.

4. Eapen M, Giralt SA, Horowitz MM, Klein JP, Wagner JE, Zhang MJ, et al. Second transplant for acute and chronic leukemia relapsing after first HLA-identical sibling transplant. Bone Marrow Transpl. 2004;34:721-7.

5. Christopeit M, Kuss O, Finke J, Bacher U, Beelen DW, Bornhäuser M, et al. Second allograft for hematologic relapse of acute leukemia after first allogeneic stem-cell transplantation from related and unrelated donors: the role of donor change. J Clin Oncol. 2013;31:3259-71.

6. Ruutu T, de Wreede LC, van Biezen A, Brand R, Mohty M, Dreger P, et al. Second allogeneic transplantation for relapse of malignant disease: retrospective analysis of outcome and predictive factors by the EBMT. Bone Marrow Transpl. 2015;50:1542-50.

7. Kharfan-Dabaja MA, Labopin M, Polge E, Nishihori T, Bazarbachi A, Finke J, et al. Association of second allogeneic hematopoietic cell transplant vs donor lymphocyte infusion with overall survival in patients with acute myeloid leukemia relapse. JAMA Oncol. 2018:4:1245-53.

8. Kobayashi S, Kanda $\mathrm{Y}$, Konuma $\mathrm{T}$, Inamoto $\mathrm{Y}$, Matsumoto $\mathrm{K}$, Uchida $\mathrm{N}$ et al. Outcomes of third allogeneic hematopoietic stem cell transplantation in relapsed/refractory acute leukemia after a second transplantation. Bone Marrow Transpl. 2021 (in press).

9. Rank A, Peczynski C, Labopin M, Stelljes M, Simand C, Helbig G, et al. Feasibility and Outcomes of a Third Allogeneic Hematopoietic Stem Cell Transplantation: A Retrospective Analysis from the Acute Leukemia Working Party of the European Society for Blood and Marrow Transplantation. Transpl Cell Ther. 2021;27:408. e1-408.e6.

10. Vago L, Perna SK, Zanussi M, Mazzi B, Barlassina C, Stanghellini MT, et al. Loss of mismatched HLA in leukemia after stem-cell transplantation. N Engl J Med. 2009;361:478-88.

11. Shimoni A, Labopin M, Finke J, Ciceri F, Deconinck E, Kröger N, et al. Donor selection for a second allogeneic stem cell transplantation in AML patients relapsing after a first transplant: a study of the Acute Leukemia Working Party of EBMT. Blood Cancer J. 2019;9:88.

\section{AUTHOR CONTRIBUTIONS}

AS wrote the manuscript.

\section{COMPETING INTERESTS}

The author declares no competing interests.

\section{ADDITIONAL INFORMATION}

Correspondence and requests for materials should be addressed to Avichai Shimoni.

Reprints and permission information is available at http://www.nature.com/ reprints

Publisher's note Springer Nature remains neutral with regard to jurisdictional claims in published maps and institutional affiliations. 